\title{
Fertilización biológica con Trichoderma spp y Azospirillum spp en maíz
}

Abarza Silvia del V.; Altamirano Fanny E.*; Zankar Graciela del C.; Boccardo Roberto J.; Britos Juan Facultad de Cs. Agrarias. Universidad Nacional de Jujuy (UNJu). Alberdi 47(4600) S.S. de Jujuy E-mail: microbio.microbio@yahoo.com.ar

Introducción:En los sistemas naturales las comunidades microbianas de la rizósfera coexisten en relativo equilibrio en procura de espacio y alimento. En un sistema agrícola este balance puede afectar las comunidades microbianas disminuyendo los organismos benéficos y beneficiando a los patógenos a niveles que pueden ser desfavorables sobre el rendimiento de los cultivos. La incorporación de microorganismos benéficos al sistema puede cambiar la estructura de la población microbiana favoreciendo la sanidad y el rendimiento de los cultivos. Asimismo los resultados de los efectos benéficos depende de la compatibilidad funcional entre los microorganismos y de las que ocurren entre los demás componentes del sistema microorganismo-planta-suelo (Cano, 2011). Los microorganismos que promueven el crecimiento de las plantas (PGPM) y los agentes de Control Biológico (CB) poseen efectos benéficos primarios y secundarios, tal es el caso de Trichoderma que juega un rol importante en el CB, pero también produce metabolitos secundarios que favorecen el crecimiento de las plantas. Los microorganismos PGPM, como Azospirillum, poseen efectos benéficos primarios y secundarios que intervienen en la promoción del crecimiento como en reducir enfermedades. Desde hace aproximadamente una década el objeto principal de nuestras investigaciones (cátedra Edafología) es introducir mejoras en el cultivo del maíz (Zea mays L.) a través de innovaciones biotecnológicas donde se contemplan los aspectos multifacéticos de los microorganismos que ayuden a reducir problemas asociados al uso de agroquímicos(Zankar et al, 2016). Por ello el propósito de este trabajo fue evaluar los efectos deTrichoderma sp.yAzospirillum sp.en el rendimiento del maíz.

Materiales y métodos: Los ensayos se realizaron en el área de los Valles Templados de Jujuy, en un lote con antecesor maíz inoculado con Azospirillum sp. La zona presenta un clima monzónico, con temperatura y precipitación media anual de $17^{\circ} \mathrm{C}$ y $621 \mathrm{~mm}$, respectivamente. El suelo corresponde a un Argiustol vértico arcilloso de la Serie Loma Atravesada. Las semillas de maízson de la variedad local "Jujeño Luna". El biofertilizante se formuló con cepas de Trichoderma sp. y Azospirillum sp. Sobre un Diseño Completamente Aleatorizado con tres tratamientos y dos repeticiones. T0: sin inocular, T1 con Trichoderma y T2 con Azospirillum. Previo a la siembra, las semillasfueron inoculadas con 0,5 ml de inoculante cada $100 \mathrm{~g}$.Se tomó una muestra de 30 mazorcas por tratamiento para evaluar rendimiento. Los datos se analizaron con ANOVA y Test DMS ( $\mathrm{p} \leq 0,05 \%)$ para comparación de medias.

Resultados: Se ha monitoreado crecimiento, sanidad y rendimiento del cultivo, observándose un buen desarrollo vegetativo y ausencia de patógenos, particularmente en T1. El análisis estadístico muestra diferencias significativas $(\mathrm{p} \leq 0,05)$ entre los tratamientos y el T0. Tanto la inoculación conAzospirillum (T2) como Trichoderma (T1) superan al T0 en $28 \%$ y $12 \%$ respectivamente,lo que indica que ambas cepas mejoran significativamente el rendimiento en maíz ( $\mathrm{Kg} / \mathrm{ha})$, siendo la diferencia entre T1 y T2 de 16\% a favor de Azospirillum. Esto sugiere que Trichoderma es esencialmente un agente de CB. En T2 se observó el efecto de Azospirillum básicamente como promotor del crecimiento. También cabe destacar que el suelo del ensayo tiene un antecesor maíz inoculado con Azospirillum donde la cepa está completamente adaptada, mientras que Trichoderma tuvo que adaptarse e interactuar con la microflora nativa.

Conclusiones: Los PGPM promueven el crecimiento y el rendimientode las plantas, pero también juegan un rol en el control de enfermedades, mientras que los agentes de CB pueden controlar enfermedades y también estimular el crecimiento de las plantas en ausencia de patógenos. Ambas funciones mejoran o sinergizan los efectos benéficos de los microorganismos de la rizósfera y la respuesta de las plantas a la inoculación depende de la compatibilidad funcional de los agentes que interactúan. Aunque aun se requieren muchos estudios para interpretar el modo de acción, las interacciones y el efecto de los microorganismos en la rizósfera, es recomendable el uso de biofertilizantes en forma controlada.

Bibliografía:

Zankar, G.; S. del V. Abarza; R. Boccardo y F. Altamirano. 2016. Inoculación múltiple en un cultivo de Poroto blanco (Phaseolus vulgaris L.) var 10. Revista Científica AGRARIA. FCA-UNJu. Año 20152016,Vol. IX, No 16, pp 62-67. ISSN 2362-4035. Disponible en: http://www.fca.unju.edu.ar

Cano, Mario Alejandro. 2011. A review of interaction of benefical microorganisms in plants: mycorrhizae, Trichoderma spp and Pseudomonas spp. Rev.U.D.C.A Act. E Div. Cient. 14(2):15-31. 